\title{
Quantum-dot heat engines with irreversible heat transfer
}

\author{
Jianying Du $\odot$, Wei Shen, Xin Zhang, Shanhe Su, ${ }^{*}$ and Jincan Chen ${ }^{\dagger}$ \\ Department of Physics, Xiamen University, Xiamen 361005, People's Republic of China
}

(Received 21 June 2019; revised manuscript received 28 September 2019; accepted 11 February 2020; published 4 March 2020)

\begin{abstract}
We study an endoreversible quantum heat engine in which the heat transfer between the baths is mediated by two qubits. Each qubit acts as an energy filter which allows for the conversion of heat into work. The relation between the efficiency and the power output is derived. It is found that the efficiency of the quantum heat engine at the maximum power output is closely dependent on the properties of quantum dots and does not equal the Curzon-Alhborn efficiency, which is only a function of the bath temperatures. The efficiency and the power output may be adjusted through qubit energy levels. It is further shown that in the limiting cases of small energy levels (or high temperatures) and small temperature differences, the quantum heat engine converges to the classical endoreversible Carnot heat engine.
\end{abstract}

DOI: 10.1103/PhysRevResearch.2.013259

\section{INTRODUCTION}

Quantization of energy and its influence on energy conversion are a part of the fundamental framework for understanding thermodynamics [1,2]. Quantum heat engines, which convert heat into useful work, are important devices to explore the thermodynamic properties of quantum systems [3]. For example, Quan et al. analyzed the thermodynamics of quantum Carnot and Otto engines utilizing harmonic oscillators, twolevel systems, and particles in an infinite square potential well as the working substance, and compared them with their classical counterparts $[4,5]$. Scully et al. revealed that the power of a quantum machine can be enhanced by noise-induced coherence [6-8]. Furthermore, quantum engines powered by nonthermal energy sources have been shown to exhibit unconventional performances [9-11].

Carnot' $s$ theorem states that all irreversible heat engines operating between two heat baths cannot attain the Carnot efficiency $\eta_{C}[12,13]$. Considering the case where the heat transfer is irreversible, Curzon and Ahlborn discovered an equation describing the efficiency of an endoreversible heat engine operating at the maximum power output, i.e., $\eta_{C A}=$ $1-\sqrt{1-\eta_{C}}$ [14], which is referred to as the Curzon-Alhborn (CA) efficiency [15-20]. This formula is applicable to many other thermodynamic machines, including Brownian heat engines [21-23], quantum-dot heat engines [24-27], low heat dissipation machines [28,29], and Feynman's ratchets [30,31], etc. Using the finite-time thermodynamics theory, Chen et al. demonstrated that $\eta_{C A}$ does not determine the upper bound of the efficiency but gives the lower bound of the optimized

\footnotetext{
*sushanhe@xmu.edu.cn

$\dagger$ jcchen@xmu.edu.cn
}

efficiency [32]. Esposito et al. recovered the CA efficiency under the symmetric dissipation for finite-time Carnot cycles [33]. Cavina et al. also recovered the CA efficiency based on a quantum Carnot cycle [34].

Several investigations regarding the small quantum thermal machines with few quantum levels or qubits have been reported [35-37]. Linden et al. researched the fundamental limitation on the size of the thermal machines [38]. Brunner et al. showed that the quantum effects are capable of enhancing energy conversion efficiency in microscopic quantum refrigerators [39]. Correa et al. proved that the coefficient of performance at the maximum cooling power for any threelevel refrigerators depends on the spatial dimensionality of the cold bath [40,41]. Levy et al. put forward a global dissipative equation for the heat transport between two qubits [42].

Despite the intensive onging developments, a quantum analog of the classical endoreversible heat engine has not yet been fully studied. The "quantumness" of the engine considered here is due to the fact that the heat transfer is described by the interaction between the quantum qubits and the heat baths rather than the classical heat-transfer laws such as the Fourier law of conduction. Taking these into account, we put forward a new model of the endoreversible quantum heat engine by considering qubits as the external heat-transfer mediums. Based on the quantum master equation approach [43-45], the influences of quantum effects on the heat currents, power output, and thermal efficiency will be analyzed. The rest of the paper is organized as follows. In Sec. II, we establish the model of a quantum heat engine with external irreversible heat transfer. In Sec. III, we illustrate the thermodynamic characteristics of the quantum heat engine. In Sec. IV, we will reveal the performances of the engine in the extreme situations. Finally, the main conclusions are drawn.

\section{MODEL AND THEORY}

Creative Commons Attribution 4.0 International license. Further distribution of this work must maintain attribution to the author(s) and the published article's title, journal citation, and DOI.
A model made up of two qubits and an endoreversible Carnot heat engine is considered here, as shown in Fig. 1. 


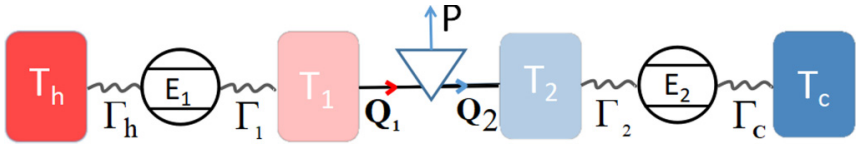

FIG. 1. The schematic diagram of a quantum endoreversible heat engine. It is made up of two qubits, 1 and 2, which interact with the harmonic thermal baths, respectively. Qubit 1 connects the hot bath and the working fluid of the engine in the isothermal process at $T_{1}$, while qubit 2 is inserted between the cold bath and the working fluid of the engine in the isothermal process at $T_{2}$. The two qubits couple with their respective reservoirs with strengths $\Gamma_{h}, \Gamma_{1}, \Gamma_{2}$, and $\Gamma_{c} . E_{1}$ and $E_{2}$ are the energy levels of qubits 1 and 2.

Qubits 1 and 2 induce, respectively, the irreversible heat fluxes $Q_{1}$ from the thermal bath $h$ to the engine and $Q_{2}$ from the engine to the thermal bath $c . T_{h}$ and $T_{c}$ are the temperatures of the high- and low-temperature thermal baths. $T_{1}$ and $T_{2}$ are the temperatures of the working fluid in two isothermal processes, which are different from the temperatures $T_{h}$ and $T_{c}$ of the thermal baths and temperatures follow the inequality, $T_{h} \geqslant T_{1} \geqslant T_{2} \geqslant T_{c}[14]$.

The free Hamiltonian of qubit 1 is given by

$$
H_{1}=\frac{E_{1}}{2} \sigma_{1}^{z},
$$

where $\sigma_{1}^{z}$ is the third Pauli operator of qubit 1 . The master equation governing the dynamics of qubit 1 reads

$$
\dot{\rho}_{1}=-i\left[H_{1}, \rho_{1}\right]+D_{h}\left[\rho_{1}\right]+D_{1}\left[\rho_{1}\right],
$$

where $\rho_{1}$ is the density matrix of qubit 1. Applying the Born-Markov and the rotating-wave approximations, one can obtain the dissipator, $D_{i}[\rho](i=h, 1)$ associated with each bath [46-49]

$$
\begin{aligned}
D_{i}\left[\rho_{1}\right]= & \Gamma_{i}\left[n_{i}\left(\sigma_{1}^{+} \rho_{1} \sigma_{1}^{-}-\frac{1}{2}\left\{\sigma_{1}^{-} \sigma_{1}^{+}, \rho_{1}\right\}\right)\right. \\
& \left.+\bar{n}_{i}\left(\sigma_{1}^{-} \rho_{1} \sigma_{1}^{+}-\frac{1}{2}\left\{\sigma_{1}^{+} \sigma_{1}^{-}, \rho_{1}\right\}\right)\right],
\end{aligned}
$$

where $\Gamma_{i}$ denotes the dissipation rate and is related to the spectral density of the bath; $\sigma_{1}^{+}=|1\rangle\langle 0|$ and $\sigma_{1}^{-}=|0\rangle\langle 1|$ are the creation and annihilation operators of qubit 1 , respectively; $n_{i}=1 /\left[\exp \left(\beta_{i} E_{1}\right)-1\right]$ is the Bose-Einstein occupation function, the inverse temperature of bath $i, \beta_{i}=1 / T_{i}$, and $\bar{n}_{i}=1+n_{i}$. In Refs. [37,43], each qubit is assumed to be described by the Boltzmann distribution. In this work, we study the irreversibile heat transfer between the bosonic baths. The stationary occupations of the qubits are written in terms of the spectral distribution of the baths. In the wide-band approximation, it can be assumed that the dissipation rate $\Gamma_{i}$ is a constant. Planck's constant $\hbar$ and Boltzmann's constant $k_{B}$ are set to be unity throughout the paper, i.e., $\hbar=k_{B}=1$. In the following discussion, the energy level $E_{i}$, temperature $T_{i}$, dissipation rate $\Gamma_{i}$, heat current $Q_{i}$, and power output $P$ will be written in the nondimensionalized form by utilizing Planck units [50].

The steady-state populations of qubit 1 can be obtained by setting the left-hand side of Eq. (2) equaling zero, i.e., $\dot{\rho}_{1}=0$, yielding

$$
\rho_{1}^{s}=\frac{1}{2}\left(1+a_{1}^{z} \sigma_{1}^{z}\right),
$$

where $a_{1}^{z}=-\frac{\Gamma_{h}+\Gamma_{1}}{\Gamma_{h} S_{h}+\Gamma_{1} S_{1}}$, and $S_{i}=n_{i}+\bar{n}_{i}$.
The heat current $Q_{h}=\operatorname{Tr}\left\{H_{1} D_{h}\left[\rho_{1}^{s}\right]\right\}$ represents the energy flowed out of bath $h$ per unit time. The heat current entered bath 1 is expressed as $Q_{1}=-\operatorname{Tr}\left\{H_{1} D_{1}\left[\rho_{1}^{s}\right]\right\}$ and $Q_{h}=Q_{1}$. Thus, the heat absorbed by the heat engine is

$$
Q_{1}=\gamma_{1} E_{1}\left(n_{h}-n_{1}\right),
$$

where $\gamma_{1}=\frac{\Gamma_{1} \Gamma_{h}}{\Gamma_{h} S_{h}+\Gamma_{1} S_{1}}$. The same computational process can be applied to qubit 2. The free Hamiltonian of qubit 2 is given by $H_{2}=\frac{E_{2}}{2} \sigma_{2}^{z}$, where $\sigma_{2}^{z}$ is the third Pauli operator of qubit 2. The heat current $Q_{2}$ removed from the heat engine is written as

$$
Q_{2}=\gamma_{2} E_{2}\left(n_{2}-n_{c}\right),
$$

where $\gamma_{2}=\frac{\Gamma_{2} \Gamma_{c}}{\Gamma_{2} S_{2}+\Gamma_{c} S_{c}}$ and the thermal occupation number $n_{j}=1 /\left[\exp \left(\beta_{j} E_{2}\right)-1\right](j=2$ or $c)$. We should note that the forms of $Q_{1}$ and $Q_{2}$ are the same as the steady-state heat flux obtained in Ref. [51], while for other types of thermal baths [52] the expression of the heat current may be different.

By using Eqs. (5) and (6), the power output $P$ and the thermal efficiency $\eta$ of the heat engine can be obtained, i.e.,

$$
\begin{aligned}
P= & Q_{1}-Q_{2} \\
= & \gamma_{1} E_{1}\left[\frac{1}{\exp \left(\beta_{h} E_{1}\right)-1}-\frac{1}{\exp \left(\beta_{1} E_{1}\right)-1}\right] \\
& -\gamma_{2} E_{2}\left[\frac{1}{\exp \left(\beta_{2} E_{2}\right)-1}-\frac{1}{\exp \left(\beta_{c} E_{2}\right)-1}\right]
\end{aligned}
$$

and

$$
\eta=\frac{P}{Q_{1}}=1-\frac{\gamma_{2} E_{2}\left(n_{2}-n_{c}\right)}{\gamma_{1} E_{1}\left(n_{h}-n_{1}\right)} .
$$

In the next section, the characteristics of the quantum heat engine with irreversible heat transfer will be discussed.

\section{EFFICIENCY AT THE MAXIMUM POWER OUTPUT}

On the basis of the analysis above, there exists irreversible heat transfer in the quantum heat engine due to the interactions between the qubits and the baths. We are interested in estimating the performance of a heat engine with external irreversibilities. Therefore, we consider an endoreversible heat engine for this quantum system. For an endoreversible heat engine, the entropy productions of the working fluid in the two isothermal processes follows the relationship

$$
\frac{Q_{1}}{T_{1}}=\frac{Q_{2}}{T_{2}} .
$$

For an endoreversible Carnot heat engine, the thermal efficiency $\eta$ is directly determined by $T_{1}$ and $T_{2}$, i.e.,

$$
\eta=1-\frac{T_{2}}{T_{1}} .
$$

By using Eqs. (8), (9), and (10), the relations between the working fluid temperatures $T_{l}(l=1,2)$ and the efficiency $\eta$ are, respectively, given by (see the Appendix)

$$
T_{1 / 2}=\frac{E_{1 / 2}}{\ln \left[\frac{1}{n_{h / c}-\gamma_{2 / 1} E_{2 / 1}\left(n_{2 / 1}-n_{c / h}\right) /\left(\gamma_{1 / 2} E_{1 / 2} \alpha_{1 / 2}\right)}+1\right]},
$$

where $\alpha_{1}=1-\eta$ and $\alpha_{2}=1 / \alpha_{1}$. 


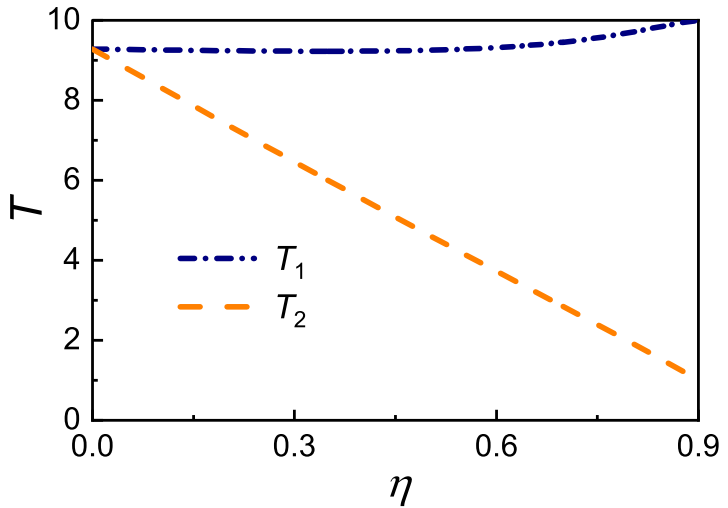

FIG. 2. The working fluid temperatures $T_{1}$ (blue dot-dashed line) and $T_{2}$ (orange dashed line) as functions of the efficiency $\eta$, where the parameters $E_{1}=10, E_{2}=6, T_{h}=10, T_{c}=1, \Gamma_{h}=\Gamma_{1}=0.01$, and $\Gamma_{2}=\Gamma_{c}=0.001$.

For a given value of $\eta$, one can obtain $T_{1}$ and $T_{2}$ with the numerical solution of the nonlinear equations based on Eq. (11). It is seen from Fig. 2 that $T_{1}$ is a monotonically increasing function of $\eta$, while $T_{2}$ is a monotonically decreasing function of $\eta$. When $\eta=0, T_{1}=T_{2}$, whose value is directly calculated from Eq. (11). When $\eta=\eta_{\max }=\eta_{C}, T_{1}=T_{h}$, and $T_{2}=T_{c}$, which is in accord with the result of a classical reversible Carnot heat engine [16].

By using Eqs. (7)-(10), the relation between the power output and the efficiency can be obtained (see the Appendix)

$$
P=\frac{\gamma_{2} E_{2} \eta}{(1-\eta)}\left\{\frac{1}{\left[\frac{1}{n_{h}-P /\left(\gamma_{1} E_{1} \eta\right)}+1\right]^{\frac{E_{2}}{E_{1}(1-\eta)}}-1}-n_{c}\right\} .
$$

Using Eqs. (5), (6), and (12), we generate the curves of the heat currents $Q_{1}$ and $Q_{2}$ and the power output $P$ varying with the efficiency $\eta$, as shown in Fig. 3, where $\eta_{P}$ is the efficiency at the maximum power output of the heat engine. It is seen from Fig. 3 that $Q_{2}$ is a decreasing function of $\eta$, while $Q_{1}$ and $P$ first increase and then decrease with the increase of $\eta$. When $\eta=0, Q_{1}=Q_{2}$ and $P=0$. When $\eta=\eta_{C}, Q_{1}=Q_{2}=0$ and $P=0$. When $\eta=\eta_{P}, P$ attains its maximum $P_{\max }$. The shape

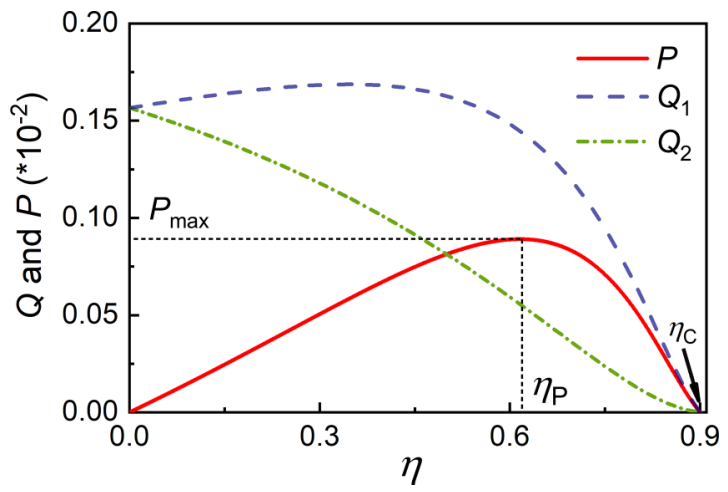

FIG. 3. The heat currents $Q_{1}$ (blue dashed line) and $Q_{2}$ (green dot-dashed line) and the power output $P$ (red solid line) as functions of the efficiency $\eta$. The values of other parameters are the same as those used in Fig. 2.
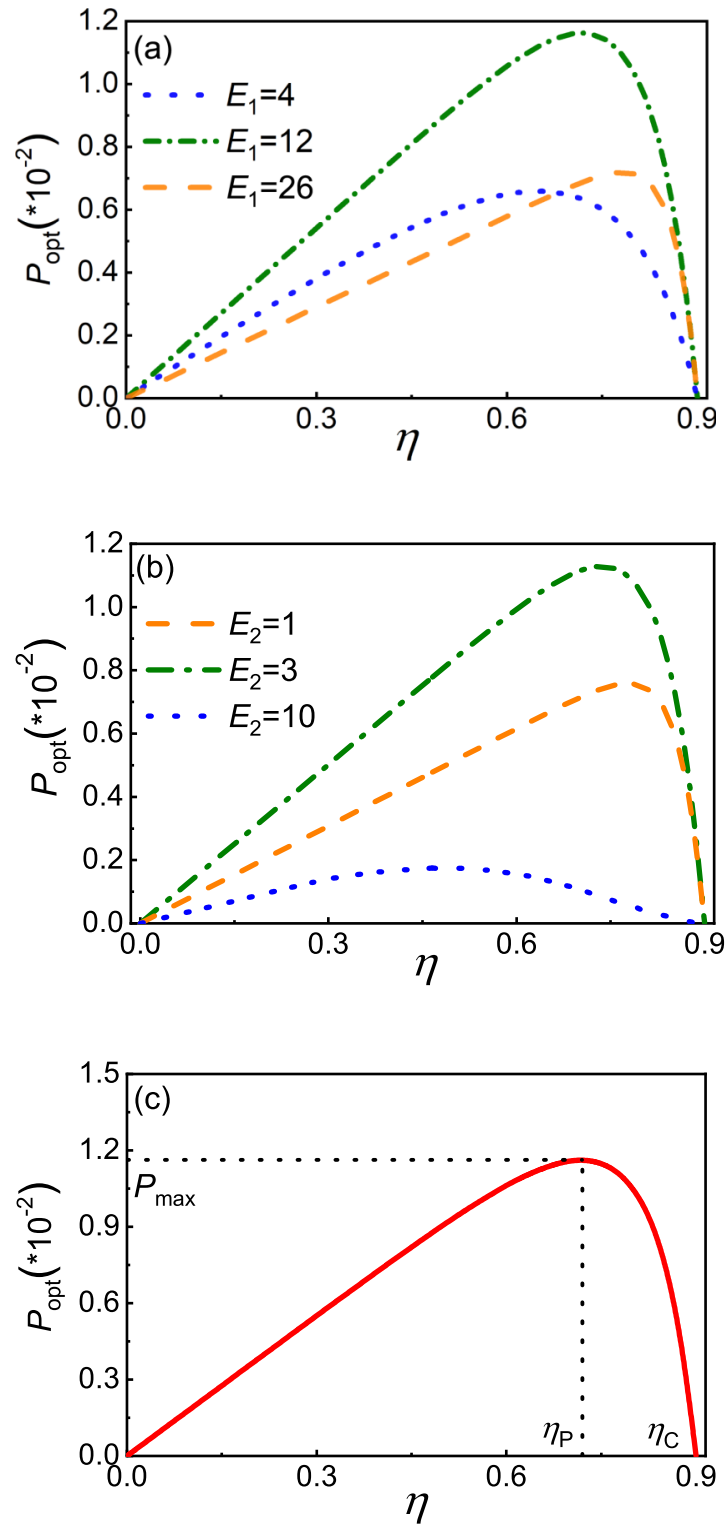

FIG. 4. The optimal curves of the power output $P_{\mathrm{opt}}$ varying with the efficiency $\eta$, where $T_{1}$ has been optimized for different values of (a) $E_{1}$ and (b) $E_{2}$. In panel (c), $E_{1}$ or $E_{2}$ has been optimized. The values of other parameters are the same as those used in Fig. 2.

of the efficiency-power characteristic curve for a quantum Carnot heat engine mentioned here is similar to that of a classical endoreversible Carnot heat engine [32]. However, the efficiency $\eta_{P}$ at the maximum power output is not equal to the CA efficiency. Obviously, both $\eta_{P}$ and $P_{\max }$ are closely dependent on the properties of qubits, as shown in Fig. 4. After the optimization with respect to $T_{1}$, Figs. 4(a) and 4(b) show the curves of the power output $P_{\text {opt }}$ varying with the efficiency $\eta$ for given values of $E_{1}$ and $E_{2}$. From Eqs. (5)-(7), it is found that the heat engine cannot generate positive power output as $E_{1}$ or $E_{2}$ moves toward zero. On the other hand, because the thermal occupation numbers in Eqs. (5) and (6) decline with the continued growth of the energy levels of qubits, the power output must be negligibly small for a very large $E_{1}$ or $E_{2}$. The $P_{\text {opt }}$ can be further optimized with respect to $E_{1}$ or $E_{2}$, 


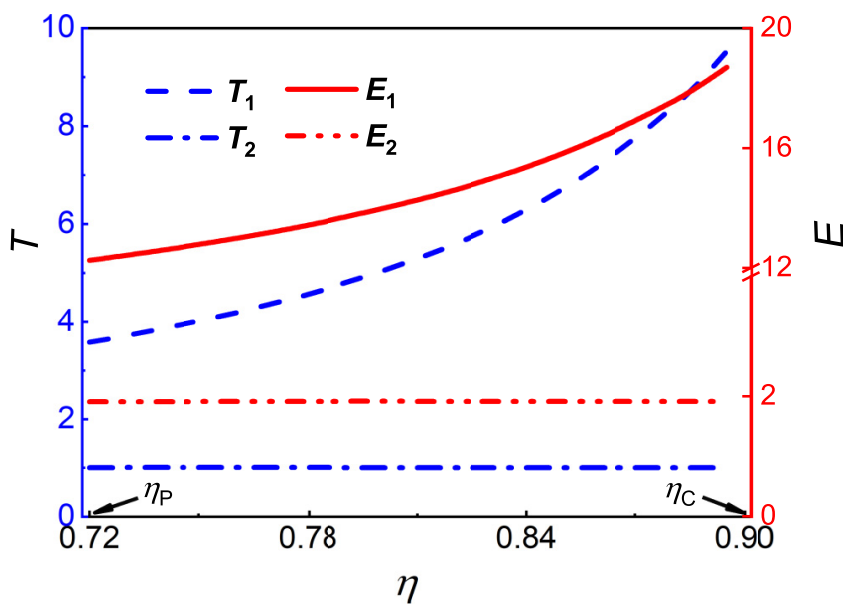

FIG. 5. In the optimal region, the temperatures $T_{1}$ and $T_{2}$ (blue lines) and the energy levels $E_{1}$ and $E_{2}$ (red lines) as functions of the efficiency $\eta$. The values of other parameters are the same as those used in Fig. 2.

as shown in Fig. 4(c). Therefore, according to Fig. 4(c), the optimal region of the efficiency should be

$$
\eta_{P} \leqslant \eta<\eta_{c}
$$

which provides the advantages in achieving high efficiency while still maintaining a large power output at the same time. Obviously, when $\eta<\eta_{P}$, the power output will decrease with the decrease of $\eta$. The quantum heat engine should be controlled so to avoid the region of $\eta<\eta_{P}$. In order for the quantum heat engine to operate in the optimal region as described by Eq. (13), the temperatures of the working fluid in the two isothermal processes must satisfy the following relations:

$$
T_{1, P} \leqslant T_{1}<T_{h}
$$

and

$$
T_{2, P} \geqslant T_{2}>T_{c},
$$

where $T_{1, P}$ and $T_{2, P}$ are, respectively, the values of temperatures $T_{1}$ and $T_{2}$ at the maximum power output. Using Eqs. (13)-(15), the relations between the two temperatures $T_{1}$ and $T_{2}$, the energy levels $E_{1}$ and $E_{2}$, and the efficiency $\eta$ in the optimal region are evaluated in Fig. 5. Both $T_{1}$ and $E_{1}$ increase with the increase of $\eta$, but $T_{2}$ and $E_{2}$ decrease with $\eta$. As $T_{1}$ increases with $\eta$, the temperature difference $T_{h}-T_{1}$ reduces, leading to the decrease of the heat current $Q_{1}$ from bath $1 . T_{1}$ continuously increases until it approaches $T_{h}$ with an increasing $\eta$. According to Eq. (5), $Q_{1}$ approaches zero for an infinitely small temperature difference $T_{h}-T_{1}$, which dramatically cuts down the power output $P$ to $P=0$. On the other hand, $T_{2}$ decreases until $T_{c}$ is reached with the increase of $\eta$. The heat $Q_{2}$ released to bath 2 can be reduced by decreasing $T_{2}$, because the temperature difference $T_{2}-T_{c}$ decreases. Further analysis shows that the power output approaches the maximum power output $P=P_{\max }$ at $\eta=\eta_{P}$. The thermal efficiency approaches the maximum efficiency $\eta=\eta_{c}$ at $T_{1} \rightarrow T_{h}$ and $T_{2} \rightarrow T_{c}$. As a result, $\eta$ is a monotonically increasing function of $E_{1}$ and a decreasing function of $E_{2}$ in the optimal region, as shown in Fig. 5.

The optimal performance of the endoreversible heat engine is analysed. According to Figs. 3, 4, and 5, the efficiency at the maximum power output is affected by the microproperties of the qubits, and, consequently, is related to the irreversible heat transfer. The energy levels $E_{l}(l=1,2)$, as the microproperties of qubits, can alter the working fluid temperatures $T_{l}$ and have significant effects on the power output $P$ and the thermal efficiency $\eta$. By adjusting the energy levels, we can regulate $P$ and $\eta$. The efficiency and the power output of the quantum heat engine can hence be adjusted through the qubit energy levels.

\section{DISCUSSION}

To better understand the performance of the quantum heat engine, we move our attention to several special situations. If $E_{l}$ is small (or the heat engine is operated at high temperatures) and $\beta_{k} E_{l}<<1(k=h, 1,2, c)$, the heat currents $Q_{1}$ and $Q_{2}$ can be rewritten as

$$
Q_{1}=\frac{1}{2} \frac{\Gamma_{1} \Gamma_{h}}{\Gamma_{1} T_{1}+\Gamma_{h} T_{h}} E_{1}\left(T_{h}-T_{1}\right)
$$

and

$$
Q_{2}=\frac{1}{2} \frac{\Gamma_{2} \Gamma_{c}}{\Gamma_{2} T_{2}+\Gamma_{c} T_{c}} E_{2}\left(T_{2}-T_{c}\right) .
$$

When $\Gamma_{1}=\Gamma_{h}, \Gamma_{2}=\Gamma_{c}, T_{h}-T_{c} \ll T_{c}, T_{h}+T_{1} \approx 2 T_{h}$, and $T_{c}+T_{2} \approx 2 T_{c}$, Eqs. (16) and (17) are simplified as

$$
Q_{1}=k_{1}\left(T_{h}-T_{1}\right)
$$

and

$$
Q_{2}=k_{2}\left(T_{2}-T_{c}\right),
$$

where $k_{1}=\frac{1}{4} \frac{\Gamma_{1} E_{1}}{T_{h}}$ and $k_{2}=\frac{1}{4} \frac{\Gamma_{2} E_{2}}{T_{c}}$. Equations (18) and (19) indicate clearly that the heat currents are proportional to the temperature differences of the working fluid and its surroundings, which obey Newton's heat transfer law $[14,16]$. This shows that when $\beta_{k} E_{l}<<1$ and the temperature difference of thermal baths is very small, the heat transfer between the engine and the thermal bath follows the classical heat transfer law. The above results indicate that the heat transfer in this irreversible system is directly affected by the value of the energy gap of qubits.

Applying Eqs. (9) and (10) and eliminating the temperatures $T_{1}$ and $T_{2}$ in Eqs. (18) and (19), one can derive the expressions of the heat current $Q_{1}$ and the power output $P$ as

$$
Q_{1}=\frac{k_{1} k_{2}}{k_{1}+k_{2}}\left[T_{h}-\frac{T_{c}}{(1-\eta)}\right]
$$

and

$$
P=\frac{k_{1} k_{2} \eta}{k_{1}+k_{2}}\left[T_{h}-\frac{T_{c}}{(1-\eta)}\right] .
$$

If the effective heat transfer coefficients $k_{1}$ and $k_{2}$ are regarded as the classical thermal conductances, Eqs. (20) and (21) exhibit the same results as obtained in Refs. [16,19]. In such a 


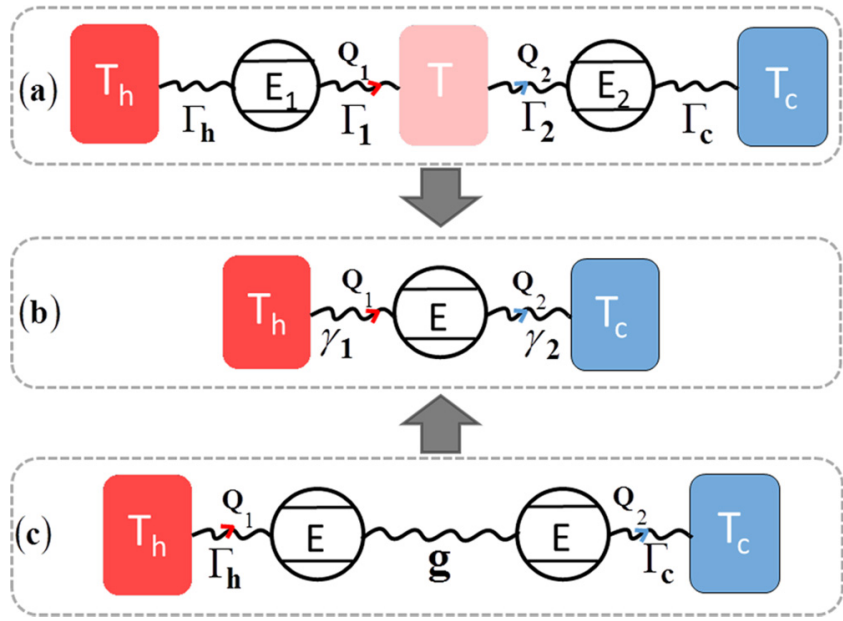

FIG. 6. The schematic diagrams of different quantum heat transfer models. (a) The two-qubit model dominated by the dissipation rates $\Gamma_{i}$. (b) The one-qubit model. (c) The two-qubit model with weak interaction $g$.

case, the efficiency at the maximum power output is equal to the CA efficiency and Eq. (13) is bounded by

$$
\eta_{C A} \leqslant \eta<\eta_{c}
$$

These results suggest that the classical correspondence of the quantum heat engine can be obtained in the limiting cases of the small energy levels of the qubits (or high-temperature heat reservoirs) and the small temperature differences of the heat reservoirs.

Finally, we discuss the equivalent conditions among the quantum heat transfer modes. When $T_{1}=T_{2}=T$, Eqs. (5) and (6) become

$$
Q_{1}=\gamma_{1} E_{1}\left(n_{h}-n_{1}\right)=\gamma_{2} E_{2}\left(n_{2}-n_{c}\right)=Q_{2},
$$

as shown in Fig. 6(a). When $E_{1}=E_{2}=E$, Eq. (23) may be further simplified as

$$
Q_{1}=\frac{\gamma_{1} \gamma_{2}}{\gamma_{1}+\gamma_{2}} E\left(n_{h}-n_{c}\right)=Q_{2},
$$

as shown in Fig. 6(b). Equations (23) and (24) show clearly that when $E_{1}=E_{2}$, Figs. 6(a) and 6(b) are equivalent to each other. This implies that the heat transferred through two qubits between the two heat reservoirs at temperatures $T_{h}$ and $T_{c}$ may be equivalent to that through one qubit operating at the same temperature difference.

It is important to note that the authors in Refs. [37,42] investigated the heat transfer in the model of quantum refrigerators and the consistency of thermodynamic laws, as shown in Fig. 6(c), where the heat current $Q_{1}$ is determined by

$$
Q_{1}=\frac{2 g^{2}\left(\Gamma_{c}+\Gamma_{h}\right) E\left(n_{h}-n_{c}\right)}{\left(\Gamma_{c}+\Gamma_{h}\right)^{2}+4 g^{2}+2 g^{2}\left(\frac{\Gamma_{c}}{\Gamma_{h}}+\frac{\Gamma_{h}}{\Gamma_{c}}\right)} .
$$

Comparing Eq. (25) with Eq. (24), we find that if the coupling strength $g$ satisfies the following relation, $g^{2}=\frac{1}{2 \gamma_{1} \gamma_{2}\left(\Gamma_{c}^{2}+\Gamma_{h}^{2}\right)}\left(\left(\gamma_{1}+\gamma_{2}\right)\left(\Gamma_{c}^{2} \Gamma_{h}+\Gamma_{h}^{2} \Gamma_{c}\right)-2 \gamma_{1} \gamma_{2} \Gamma_{c} \Gamma_{h}+\right.$ $\left\{\Gamma_{c} \Gamma_{h}\left[-2 \gamma_{1}^{2} \gamma_{2}^{2}\left(\Gamma_{c}+\Gamma_{h}\right)^{2}\left(\Gamma_{c}^{2}+\Gamma_{h}^{2}\right)\right]+\Gamma_{c} \Gamma_{h}\left[\gamma_{2}\left(\Gamma_{c}+\Gamma_{h}\right)\right.\right.$ $\left.\left.\left.+\gamma_{1}\left(\Gamma_{c}+\Gamma_{h}-2 \gamma_{2}\right)\right]^{2}\right\}^{\frac{1}{2}}\right)$, Fig. 6(c) is equivalent to Fig. 6(b).
This shows clearly that when $E_{1}=E_{2}=E$ and the coupling strength $g$ is suitably adjusted, the three heat transfer modes in Figs. 6(a), 6(b), and 6(c) are equivalent to each other. These results not only allow us to simplify complex thermodynamical systems but also provide a new perspective from which to understand the quantum heat engines.

\section{CONCLUSIONS}

A novel heat engine model including two qubits has been established and the performance characteristics of the quantum heat engine as affected by irreversible heat transfers are evaluated. It is found that the heat transfer mode between the heat engine and the heat reservoir not only depends on the properties of the qubits but also is affected by the temperatures of the heat reservoirs and their temperature differences. The power output and the efficiency can be regulated by adjusting the energy levels of the qubits. This is very different from the cases of classical heat engines. For general cases $[53,54]$, the efficiency at the maximum power output is not equal to the CA efficiency, which is usually derived under different approximations. For the limiting cases of small energy levels of qubits and small temperature differences, the quantum Carnot heat engine is equivalent to the endoreversible Carnot heat engine that obeys Newton's heat transfer law.

\section{ACKNOWLEDGMENTS}

This work has been supported by the National Natural Science Foundation of China (Grant No. 11805159), the Fundamental Research Fund for the Central Universities (No. 20720180011), and the Natural Science Foundation of Fujian Province (No. 2019J05003).

\section{APPENDIX: DETAILED DERIVATION OF EQS. (11) AND (12)}

In this Appendix, we first prove the expression of $T_{1}$ and $T_{2}$ in Eq. (11) by using Eqs. (8)-(10), and then derive the relation between the power output $P$ and the efficiency $\eta$ [Eq. (12)].

Starting from Eq. (8) and the definition of $n_{k} \quad(k=$ $h, 1,2, c)$, one finds

$$
\frac{\gamma_{2} E_{2}\left(n_{2}-n_{c}\right)}{\gamma_{1} E_{1}(1-\eta)}=n_{h}-\frac{1}{\exp \left[\beta_{1} E_{1}\right]-1} .
$$

As a result,

$$
\beta_{1} E_{1}=\ln \left\{\frac{1}{n_{h}-\gamma_{2} E_{2}\left(n_{2}-n_{c}\right) /\left[\gamma_{1} E_{1}(1-\eta)\right]}+1\right\} .
$$

Since $\beta_{1}$ is the inverse temperature of bath $T_{1}$,

$$
T_{1}=E_{1} / \ln \left[\frac{1}{n_{h}-\gamma_{2} E_{2}\left(n_{2}-n_{c}\right) /\left(\gamma_{1} E_{1} \alpha_{1}\right)}+1\right] .
$$

Similarly, $T_{2}$ can also be obtained:

$$
T_{2}=E_{2} / \ln \left[\frac{1}{n_{c}-\gamma_{1} E_{1}\left(n_{1}-n_{h}\right) /\left(\gamma_{2} E_{2} \alpha_{2}\right)}+1\right] .
$$

Combining Eqs. (A3) and (A4), we have Eq. (11). 
According to the expressions of the heat currents [Eqs. (5) and (6)],

$$
T_{1}=E_{1} / \ln \left[\frac{1}{n_{h}-Q_{1} /\left(\gamma_{1} E_{1}\right)}+1\right]
$$

and

$$
T_{2}=E_{2} / \ln \left[\frac{1}{n_{c}+Q_{2} /\left(\gamma_{2} E_{2}\right)}+1\right] .
$$

Combing Eq. (A5) with Eq. (A6), we immediately have

$$
1-\eta=\frac{E_{2} / \ln \left[\frac{1}{\left.\frac{1}{n_{c}+Q_{1}(1-\eta) /\left(\gamma_{2} E_{2}\right)}+1\right]} .\right.}{E_{1} / \ln \left[\frac{1}{n_{h}-Q_{1} /\left(\gamma_{1} E_{1}\right)}+1\right]} .
$$

Using Eq. (A7) and the expression of the power output $P=$ $Q_{1} \eta$, we arrive at Eq. (12).
[1] J. P. Pekola, Nat. Phys. 11, 118 (2015).

[2] F. Binder, S. Vinjanampathy, K. Modi, and J. Goold, Phys. Rev. E 91, 032119 (2015).

[3] S. Su, J. Chen, Y. Ma, J. Chen, and C. Sun, Chin. Phys. B 27, 060502 (2018)

[4] H. T. Quan, Y.-x. Liu, C. P. Sun, and F. Nori, Phys. Rev. E 76, 031105 (2007).

[5] H. T. Quan, Phys. Rev. E 79, 041129 (2009).

[6] M. O. Scully, M. S. Zubairy, G. S. Agarwal, and H. Walther, Science 299, 862 (2003).

[7] M. O. Scully, K. R. Chapin, K. E. Dorfman, M. B. Kim, and A. Svidzinsky, Proc. Natl. Acad. Sci. USA 108, 15097 (2011).

[8] K. E. Dorfman, D. V. Voronine, S. Mukamel, and M. O. Scully, Proc. Natl. Acad. Sci. USA 110, 2746 (2013).

[9] W. Niedenzu, V. Mukherjee, A. Ghosh, A. G. Kofman, and G. Kurizki, Nat. Commun. 9, 165 (2018).

[10] X. L. Huang, T. Wang, and X. X. Yi, Phys. Rev. E 86, 051105 (2012).

[11] J. Klaers, S. Faelt, A. Imamoglu, and E. Togan, Phys. Rev. X 7, 031044 (2017).

[12] M. Polettini, G. Verley, and M. Esposito, Phys. Rev. Lett. 114, 050601 (2015).

[13] B. Lin and J. Chen, Phys. Rev. E 67, 046105 (2003).

[14] F. L. Curzon and B. Ahlborn, Am. J. Phys. 43, 22 (1975).

[15] I. I. Novikov, At. Energy 3, 1269 (1957).

[16] J. Chen, J. Phys. D 27, 1144 (1994).

[17] E. Açıkkalp, Energy Convers. Manage. 86, 792 (2014).

[18] J. Chen and Z. Yan, J. Phys. D 26, 1581 (1993).

[19] L. Chen and Z. Yan, J. Chem. Phys. 90, 3740 (1989).

[20] Z. Yan and J. Chen, J. Chem. Phys. 92, 1994 (1990).

[21] T. Schmiedl and U. Seifert, Europhys. Lett. 81, 20003 (2007).

[22] P. Hänggi and F. Marchesoni, Rev. Mod. Phys. 81, 387 (2009).

[23] Z. C. Tu, Phys. Rev. E 89, 052148 (2014).

[24] M. Esposito, R. Kawai, K. Lindenberg, and C. Van den Broeck, Phys. Rev. E 81, 041106 (2010).

[25] M. Josefsson, A. Svilans, and A. M. Burke, Nat. Nanotechnol. 13, 920 (2018).

[26] M. Esposito, K. Lindenberg, and C. Van den Broeck, Europhys. Lett. 85, 60010 (2009).

[27] B. De and B. Muralidharan, Phys. Rev. B 94, 165416 (2016).

[28] S. Bilal and R. Ramaswamy, Phys. Rev. E 87, 034901 (2013).

[29] J. Xu and C. P. Wong, Appl. Phys. Lett. 87, 082907 (2005).

[30] S. Velasco, J. M. M. Roco, A. Medina, and A. C. Hernández, J. Phys. D 34, 1000 (2001).
[31] C. Z. Tu, J. Phys. A 41, 312003 (2008).

[32] J. Chen, Z. Yan, and G. Lin, Energy Convers. Manage. 42, 173 (2001).

[33] M. Esposito, R. Kawai, K. Lindenberg, and C. Van den Broeck, Phys. Rev. Lett. 105, 150603 (2010).

[34] V. Cavina, A. Mari, and V. Giovannetti, Phys. Rev. Lett. 119 , 050601 (2017).

[35] O. Abah and M. Paternostro, Phys. Rev. E 99, 022110 (2019).

[36] G. Guarnieri, G. T. Landi, S. R. Clark, and J. Goold, Phys. Rev. Res. 1, 033021 (2019).

[37] J. Y. Du and F. L. Zhang, New J. Phys. 20, 063005 (2018).

[38] N. Linden, S. Popescu, and P. Skrzypczyk, Phys. Rev. Lett. 105, 130401 (2010).

[39] N. Brunner, M. Huber, N. Linden, S. Popescu, R. Silva, and P. Skrzypczyk, Phys. Rev. E 89, 032115 (2014).

[40] L. A. Correa, J. P. Palao, D. Alonso, and G. Adesso, Sci. Rep. 4, 3949 (2015).

[41] L. A. Correa, J. P. Palao, G. Adesso, and D. Alonso, Phys. Rev. E 87, 042131 (2013).

[42] A. Levy and R. Kosloff, Europhys. Lett. 107, 20004 (2014).

[43] N. Brunner, N. Linden, S. Popescu, and P. Skrzypczyk, Phys. Rev. E 85, 051117 (2012).

[44] C.-s. Yu and Q.-y. Zhu, Phys. Rev. E 90, 052142 (2014).

[45] A. S. Trushechkin and I. V. Volovich, Europhys. Lett. 113, 30005 (2016).

[46] H. Breuer and F. Petruccione, The Theory of Open Quantum Systems (Oxford University Press, New York, 2002).

[47] D. Walls and G. Milburn, Quantum Optics (Springer, Berlin, 1994).

[48] G. Lindblad, Commun. Math. Phys. 48, 119 (1976).

[49] V. Gorini, A. Kossakowski, and E. C. G. Sudarshan, J. Math. Phys. 17, 821 (1976).

[50] J. D. Barrow, The Constants of Nature, from $\alpha$ to $\omega$ : The Numbers that Encode the Deepest Secrets of the Universe (Pantheon, New York, 2002).

[51] D. Segal and A. Nitzan, Phys. Rev. Lett. 94, 034301 (2005).

[52] L. A. Wu, C. X. Yu, and D. Segal, Phys. Rev. E 80, 041103 (2009).

[53] V. Cavina, A. Mari, A. Carlini, and V. Giovannetti, Phys. Rev. A 98, 012139 (2018).

[54] Y. Apertet, H. Ouerdane, C. Goupil, and P. Lecoeur, Phys. Rev. E 85, 041144 (2012). 